\title{
A Resource Management System Based on the ODP Trader Concepts and X.500*
}

\author{
A Warren Pratten, James W. Hong, Michael A. Bauer \\ J. Michael Bennett and Hanan Lutfiyya \\ Department of Computer Science \\ University of Western Ontario \\ \{warren, jwkhong, bauer, mike, hanan\} @csd. uwo.ca
}

\begin{abstract}
Distributed computing systems are composed of various types of hardware and software resources. Providing a reliable and efficient distributed computing environment largely depends on the effective management of these resources and the services that they provide. ISO has begun work on a proposed standard for Open Distributed Processing (ODP). The ODP framework includes a mechanism called the Trader which provides a framework for exchanging services in an open distributed computing environment. This paper presents a design of a resource information management system which employs and extends the ODP Trader concepts to facilitate the management and use of resources, information about resources and the services provided by the resources. We describe the architecture, information model, and user interface of the resource management system. We also describe a prototype implementation which uses the X.500 Directory Service as its repository for resource information and report on our experience with it to date.
\end{abstract}

[Keywords: distributed resource management system, ODP Trader, X.500 Directory Service, information repository, distributed computing resources]

\section{Introduction}

The trend of computing in the 90's is towards distributed computing. Computing systems, which are geographically dispersed, are interconnected through communications networks and cooperate to achieve intended tasks. Such computing systems are composed of a variety of hardware and software resources. Some of these resources are static, such as devices and others are dynamic, like servers which may come and go as demand dictates. As the size and heterogeneity of these computing systems increase, so too will the number and type of resources. Since users of these systems

\footnotetext{
${ }^{*}$ This research work is supported by the IBM Center for Advanced Studies and the Natural Sciences and Engineering Research Council of Canada.
} 
depend on these resources, the effective and efficient use of these resources will be critical. An essential prerequisite of such use and sharing is the management of the various distributed resources, including keeping track of what resources are available, where they are located, what their properties are, what their statuses are, etc. Management of resources also includes maintaining similar information about the services that the resources provide. This is especially important in a distributed environment where systems come and go, servers are migrated or replicated, etc.

Resource management has always been a primary concern in centralized computing environments and operating systems. However, managing resources is much simpler in centralized systems than in distributed systems, since the resources are confined to a single location and, in general, the operating system has full control of them. In distributed computing systems, these resources are scattered throughout the distributed computing environment and no single entity has full control of these resources. Thus, the management of resources and there associated services in a distributed computing environment is inherently more difficult. As part of our work into services and tools to help manage a distributed computing environment $[1,7]$, we have looked into problems associated with the management of resources, information about the resources and their services.

ISO has begun work on a proposed standard for Open Distributed Processing (ODP) [10]. Included in this proposed standard is a mechanism called the Trader, which provides a framework for "trading" services in an open distributed computing environment [11]. "Trading" is an ODP term that is defined as the sharing of services between ODP entities (or objects). The ODP framework (including the Trader) has been continuously going through design and refinement stages and no implementation of the ODP environment currently exists. Although there has been some work on the refinement of the Trader [3,9] and investigation of the potential uses in distributed computing environments $[12,15]$, more work is required for it to become an acceptable international standard.

Our interest in the ODP Trader is motivated by several goals. First, we required a resource information management facility as part of our work investigating distributed systems management services[8]. We feel that the ODP Trader can be a good candidate to support such a management facility to maintain and provide information about resources and their services. Second, we believe that a functional component such as the Trader will be an essential component in a distributed computing environment and thus requires further research in its role, use, and interoperability with other components. Ultimately, our aim is to communicate our experiences (both design and implementation) with the Trader to the developers and users of the ODP framework.

In this paper, we present a design of a resource information management system. The aim of the system is to help manage and facilitate use of resources, information about resources and their services in a distributed computing environment. Our motivation is to use such a system to support a variety of management activities, but it can also be used to support applications and users in general. The design of the information management system is based on the ODP Trader and, hence, we refer to it as the Trader-Based Resource Management System (TBRMS). We present an architecture of TBRMS and its major components. We also describe a prototype implementation of TBRMS, which uses the X.500 Directory Service [5, 6] as its repository for resource information.

The rest of the paper is organized as follows. In Section 2, we provide a brief overview of ODP Trader. Section 3 discusses general requirements for a resource management system in a distributed computing environment. Section 4 presents a design for the Trader-Based Resource Management System. Section 5 describes our implementation effort of a TBRMS prototype using the X.500 Directory Service. Our experience with it to date is provided in Section 6. We summarize our work and discuss possible future work in Section 7. 


\section{Overview of the ODP Trader}

The ODP is a set of draft standard documents $[10,11]$ that are aimed at a variety of architectures, networks, and operating systems to provide an open distributed processing environment. The ODP Trader is one component of the ODP environment. The Trader's purpose is to provide a matchmaking facility between ODP objects.

The real advantage of the ODP Trader is in large distributed environments where objects need to be made aware of the services available. The Trader allows ODP objects to be configured into an ODP environment without prior knowledge of the services or service providers within that environment. The Trader allows this by acting as a third party that enables the dynamic service selection and the linking of clients and servers.

The ODP Trader document [11] discusses a large number of components that will comprise the Trader. Some of these deal specifically with trading policies, security requirements, accounting requirements, transfer requirements, quality of service, and federation. However, for our purposes we are looking at starting with a minimal set of functions that can later be extended to handle other concerns.

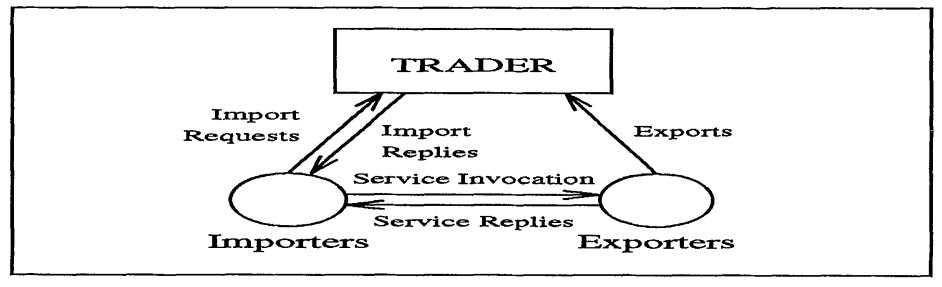

Figure 1: ODP Trader and its Clients

At the core of the ODP Trader system are the interactions among four different types of objects: traders, importers, exporters, and services (see Figure 1). An exporter is an ODP term for a service provider. It is an object with a service that it wishes to make available to other objects. Providing a service is accomplished by exporting the service to the Trader. An exporter is also able to later withdraw (e.g., make unavailable) the service. In ODP terminology, a requester of services is known as an importer. The expectation is that importers in the ODP environment can operate without any prior knowledge of where the required services are or which object provides them. To find these services the importer must make a service request to the Trader. The Trader then returns to the importer the details of the services matching the service request if any exist. A service is a function provided by an exporter for use by other ODP objects. A service may be one of the following types: an atomic operation (e.g., write), a sequence of operations (e.g., open, write, close), or a set of operations (e.g., read, write, open, close).

A service is exported in the form of a service offer which describes the service being made available. An importer discovers services by sending import requests to the Trader. The main component of an import request is the service request, which is a set of assertions that describes the desired service. The import request also provides information describing the method and scope of the search to be used by the Trader. 
It is the purpose of the Trader to match the service requests of the importers with the service offers of the exporters. This is done by matching the assertions in the service request with the assertions that compose the service properties of the offered services. The Trader sends to an importer the details of the services (including location) that match its service requirements.

\section{TBRMS Requirements}

We have based our TBRMS design upon three primary requirements: providing a functional architecture for the TBRMS, providing a simple set of service interfaces, and employing a repository for storing resource information.

\subsection{Functional Architecture}

A certain level of functionality will be needed to exist within the TBRMS to adequately respond to client requests. The TBRMS will need components to communicate with the clients, parse their requests, and provide a means through which resource information may be stored, retrieved, updated, and deleted. The TBRMS should also offer some means of assuring the status of resources for which it is responsible and a method of controlling client access to the resource information.

The TBRMS architecture should be clean, extensible, and modularized. It should allow the delegation of tasks to various subcomponents in such a way that the requests of the clients are dealt with in a logical, coordinated, and timely fashion.

\subsection{Service Interfaces}

The TBRMS service interfaces should provide simple access to the TBRMS for the TBRMS clients. Three types of interactions will be involved between the clients (or users) and the TBRMS and thus appropriate interfaces should be provided to support them.

General Client Interactions: All clients will need a method of establishing an association with the TBRMS and later breaking that association when the TBRMS service is no longer required.

Exporter Interactions: The TBRMS must provide interfaces through which an exporter may add, change, or remove any description of resources it wishes to make available to other TBRMS clients.

Importer Interactions: The TBRMS must provide a method which allows an importer to do resource discovery based on its resource requirements and the resource descriptions maintained by the TBRMS.

\subsection{Resource Information Repository}

The very nature of the TBRMS requires that a resource information repository that stores resource information form a crucial element of our TBRMS design. Some of the necessary characteristics of the resource information repository are: extensible data modeling capabilities, general naming scheme, distributed service, heterogeneous data sources, good performance, and security [7]. 


\section{Design of TBRMS}

In this section, we present a design for the Trader-Based Resource Management System. We describe the architecture of TBRMS as well as its service interfaces.

\subsection{TBRMS Architecture}

Our TBRMS architecture defines the major components that interact to function as the TBRMS. These components are TBRMS Coordinator, Request Parser, Access Control, Inventory Control, Matcher, Resource Information Maintainer, and Federator. Figure 2 illustrates the TBRMS architecture.

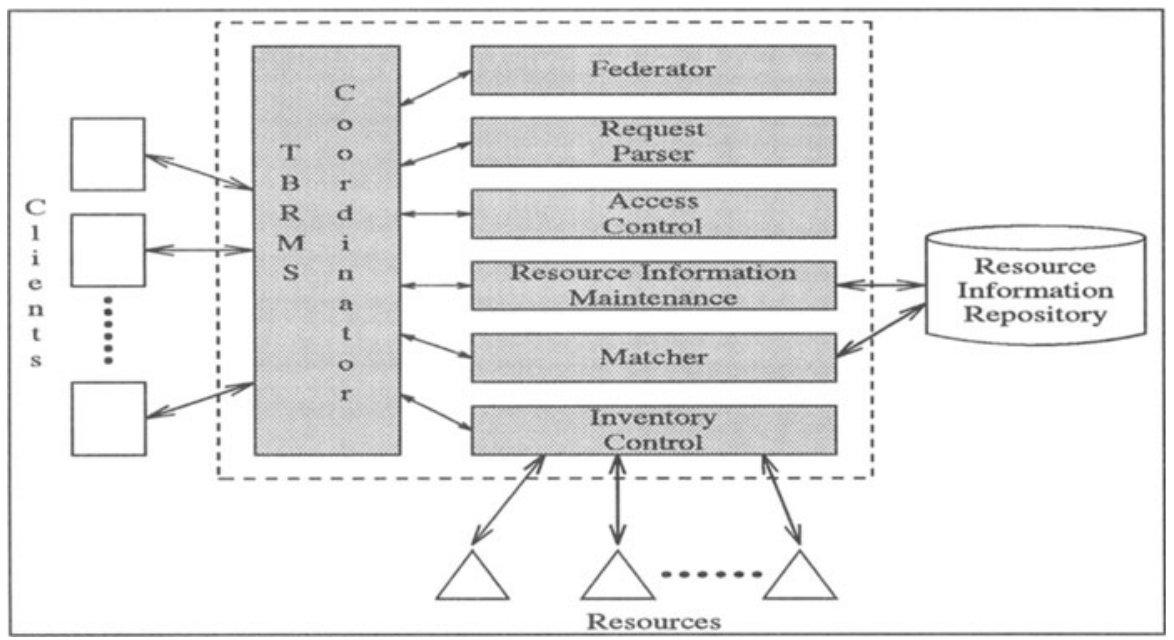

Figure 2: TBRMS Architecture

TBRMS Coordinator: This component coordinates activities within the TBRMS and acts as a front end to the TBRMS. As client requests are received by the TBRMS, the Coordinator acts upon them by interacting with the other TBRMS components. It coordinates the activities within the TBRMS to produce timely responses to client requests.

Request Parser: This component takes the client requests and translates them into an internal format which will later be translated into requests of the type understood by the Resource Information Repository.

Access Control: This component is used to determine the extent to which clients may make use of the TBRMS. For example, an importer must be registered with the TBRMS before it may request resources, and a client must be the owner (exporter) of a resource to modify or withdraw it. 
Inventory Control: This component is used to interact with resources to enquire about their status, including determining whether a resource is still up and running.

Resource Information Maintenance: This component exists to provide an interface to the Resource Information Repository. It provides the functionality that allows the TBRMS to

- add new information on resources

- delete information on resources

- modify information on resources

- list available resources

- search for specific resources

Matcher: This component queries the Resource Information Repository for resources. The queries are generated by the Request Parser component based on the resource requests of a client. The Matcher returns all resources matching the original request.

Federator: To be effective in a distributed environment the TBRMS should not be a centralized service but should instead be distributed in some manner. The Federator component provides the means by which two TBRMSs could communicate to share the resources each manages with the other. The Federator component in part determines which resources may be shared with another TBRMS. The ODP Trader document [11] describes the federation (or interworking) of Traders which other work has examined $[2,13,17]$.

\subsection{TBRMS Service Interfaces}

The service interfaces of the TBRMS system represent points of interactions between the TBRMS and its clients. These interfaces have been grouped by function, namely client, importer, and exporter. The details of the interface specifications can be found in [14].

\subsubsection{Client}

Before any client (importer or exporter) may make use of the TBRMS we require that the client first register with the TBRMS. Accordingly, when a client is finished making use of the TBRMS, we require that the client deregister itself. Although strictly speaking this set of interfaces is not necessary for a working TBRMS, we felt that there should exist some method by which the TBRMS could keep track of its clients. Forcing clients to register before using the TBRMS allows the TBRMS to have knowledge of its clients. This will become more important with security extensions to the TBRMS.

register: The operation called register allows a client to register itself with a TBRMS. Since a client may use the TBRMS to both import and export resources there is no need for the client to state what use it will make of the TBRMS.

deregister: The operation called deregister allows a client to deregister itself from a TBRMS. 


\subsubsection{Importer}

Importers are TBRMS clients which have resource requirements that need to be fulfilled. The set of importer operations provide a method that allows a client to do some resource discovery and eventually provide the information necessary to reference a particular resource.

search: The operation called search can be used by an importer to discover the resources matching a set of resource requirements. The matching criteria is an expression using attribute-based matching to represent the resource requirements of the importer. The TBRMS returns to the client references for those resources matching its stated requirements.

list: The operation called list is used by an importer to retrieve the details of a particular resource. A client may use the list operation on a variety of resources to select the most appropriate resource to fulfill its resource needs. An importer client uses the previously acquired resource identifier for the resource of interest.

select: The operation called select is used by an importer client to retrieve the interface to a resource. The client must supply a previously obtained resource identifier.

\subsubsection{Exporter}

Exporters are TBRMS clients which have resources they are willing to make available to other clients in the distributed system. Although the exporter allows other processes to use its resources, the exporter maintains control of the resource and may change or withdraw the resource at its convenience.

export: The operation called export is used by an exporter wishing to make a resource available through the TBRMS. The exporting client supplies to the TBRMS the resource properties for a resource. The resource properties are expressed as a list of assertions about the resource.

withdraw: The operation called withdraw is used by an exporter which, after previously exporting a resource, now wishes to remove the reference of the resource from the TBRMS. Note that withdrawing a resource is not necessarily equivalent to deleting or killing the resource. It simply removes the resource from the TBRMS, restricting any new usage by other clients.

update: The operation called update is used by an exporter which, after previously exporting a resource, now wishes to update some or all values associated with that resource; for example an exporter may want to change the values associated with the attributes queueLength and costPerPage for an exported printer resource. Strictly speaking this operation could be accomplished by the sequence of withdrawing the resource and then exporting the resource with the updated information, but one advantage of allowing updates is that the resource retains its resource identifier.

\subsubsection{Status Responses}

It is a basic assumption of the TBRMS system that the clients may rely on the TBRMS being in good working order. This is true because clients might depend on the TBRMS to provide essential services. Therefore it is important that the clients receive from the TBRMS messages indicating the status of their operations on the TBRMS interfaces. Examples of status responses would be Ok, clientUnknown and resourceNotFound. 


\section{TBRMS Prototype Implementation}

A prototype Trader-Based Resource Management System has been developed to demonstrate that the TBRMS provides a viable means where by resources may be managed in a distributed computing environment. In this section, we present the details behind the TBRMS prototype implementation.

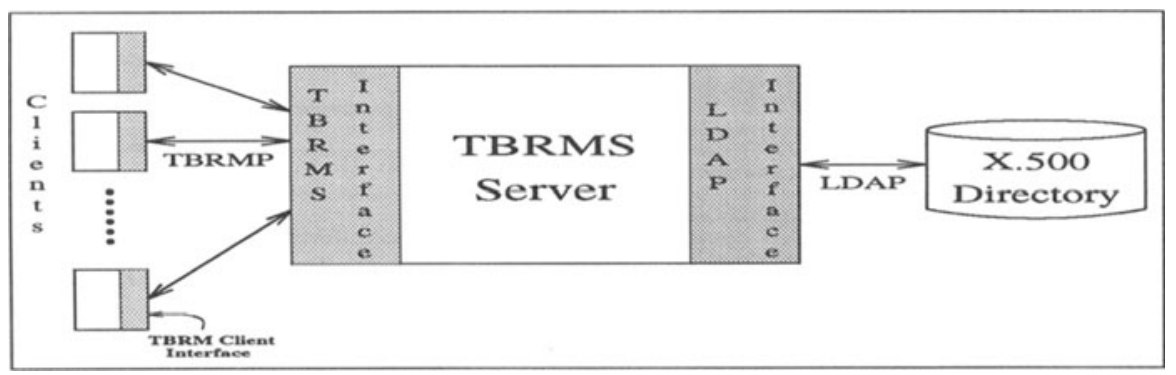

Figure 3: Prototype Implementation of TBRMS Architecture Using X.500

Figure 3 illustrates the architecture of the TBRMS prototype which is based on the TBRMS architecture described in Section 2. Work with the prototype has taken place within the UWOCSD Systems Lab. This lab is comprised of a network of heterogeneous computers consisting of Sun Sparc, Sun 3, IBM RS6000 and MIPS workstations as well as a 10-processor Sequent Symmetry.

The prototype TBRMS server runs on one of the Sun Sparc workstations. Clients running on all system lab machines have successfully interacted with the prototype TBRMS server. The clientTBRMS communication is provided by the Trader-Based Resource Management Protocol [14] which was implemented using the Open Network Consortium's (ONC) Remote Procedure Call mechanism [4]. The TBRMS Service Interfaces described in Section 4.2 are mapped onto the operations offered by the TBRMS.

The prototype relies on the X.500 Directory Service [5, 6] as its resource information repository. The X.500 Directory Service possesses some essential properties that satisfy the requirements of our resource information repository, in particular its powerful information modelling capability, global naming scheme, distributed service, and simple access interface $[7,18]$. The X.500 Directory contains entries (or objects) which describe information about entities (e.g., resources). An objectoriented approach is used for modelling directory information objects and allows the users to define any information object class by either extending existing classes or defining entirely new classes.

The prototype TBRMS uses the ISODE Quipu 8.0 implementation of X.500 [16] and a directory service agent (DSA) running on a second Sun Sparc workstation within the lab. The TBRMS accesses the DSA through the light-weight directory access protocol (LDAP) [19].

At present, the prototype TBRMS only does a weak form of access control. Each client and resource is assigned a unique identifier which is used in any subsequent interaction with the TBRMS. Authentication is performed using this identifier to ensure a client has the ability to perform its requested actions. For example a check is made before a client is allowed to update or withdraw a resource. Currently all authentication is carried out by performing search and read operations on the X.500 directory information. That is, when a client makes a request the TBRMS uses the iden- 
tifier provided by the client to search the directory. If an entry with a matching identifier is found the client is assumed to be valid. Similarly if the request involves either withdrawing or updating a resource then the operation is allowed only if the directory entry contains both the client's and resource's identifiers.

The actual resource types were implemented using X.500's object classes [14]. This provides a good method of ensuring type checking on resource definitions. When a resource is exported one of its attributes must be a resourceType. The value associated with the resource type is used as part of the X.500 object class.

\section{Experience}

To demonstrate the functionality of the TBRMS we show how a sample client-server application has been modified to use the TBRMS. The application is a locally developed password maintenance system. The password maintenance system consists of one password server (or daemon) program (passwdd) and multiple password client programs (passwd) running throughout the distributed computing environment in the Department of Computer Science. This password maintenance system provides the ability for users to change their passwords from remote machines. Typically one machine acts as the server for a domain and access to the server is limited to password clients within that domain. Whenever a user within the domain wishes to change his/her password, they use the local password client program which connects with the password daemon and changes the user's password on their behalf. Figure 4 illustrates this password maintenance system.

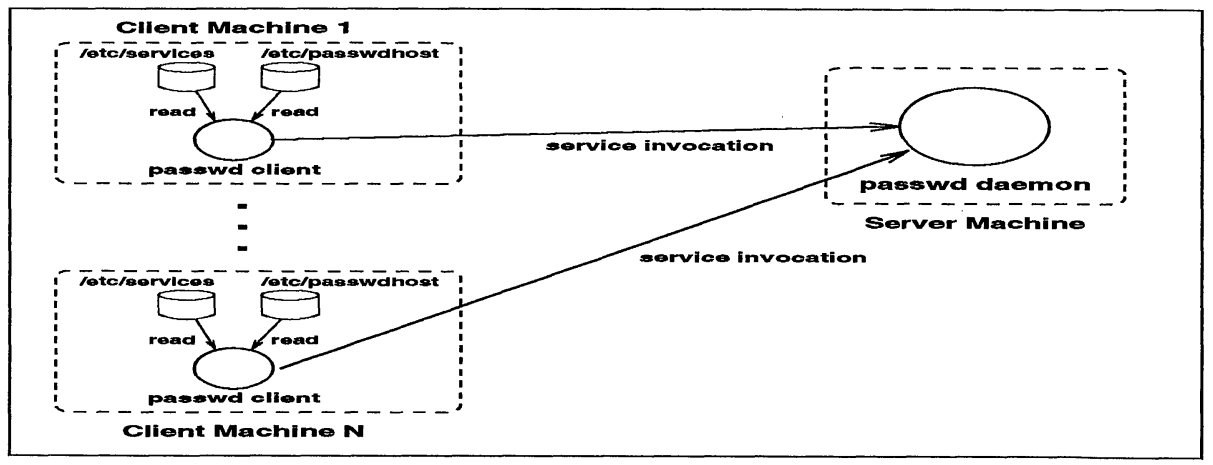

Figure 4: The Password Maintenance System

In order for the client to contact the password daemon, the client must have some way of locating the daemon. The original version of the password client reads two different files to locate the daemon. The first file (/etc/passwdhost) tells the client which machine is running the daemon. The other file (/etc/services) tells the client which port on that machine the daemon is listening to. Both these files remain relatively static, meaning that if the daemon is moved to a new machine the /etc/passwdhost and /etc/services files on all client machines would need to be updated by hand. 


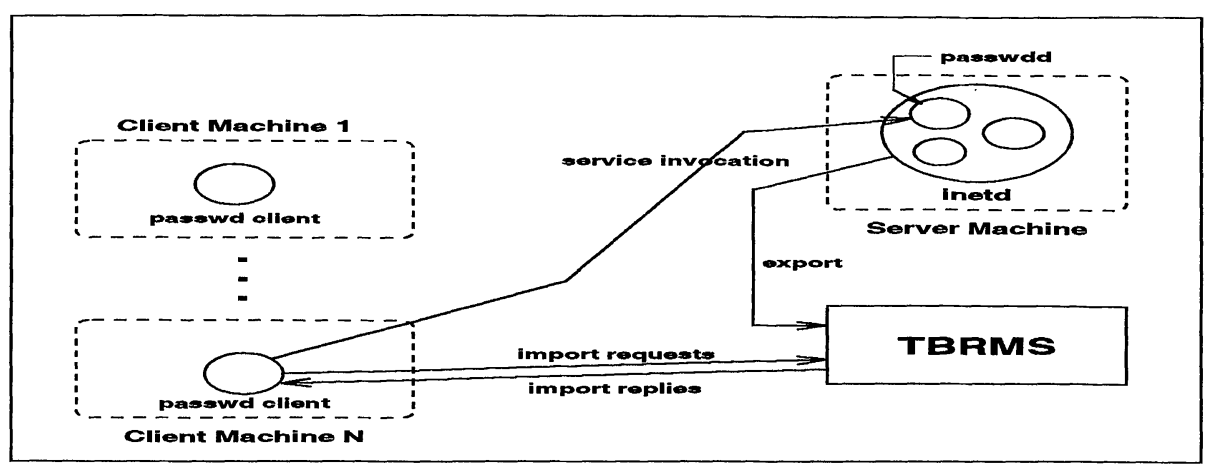

Figure 5: The Password Maintenance System using TBRMS

Using the TBRMS simplifies locating the password maintenance service in the network. Figure 5 illustrates the new password maintenance system using TBRMS. For the purposes of our previous discussion we could view the password daemon program as being an independent server. In actual fact access to the password daemon is controlled by an Internet services daemon called inetd which is responsible for invoking the password daemon when a client contacts the appropriate service port.

Another way of viewing inetd is as a service provider and passwdd is one of the services it offers. The resource type tbrminetdService was defined to describe the services offered by inetd. Since the services offered by inetd are a resource sub-type of the more general tbrmGeneralResource type we can specify the tbrmGeneralResource in our definition for tbrmlnetdService and then only specify the new attributes that define the new resource type.

Using the TBRMS with the password maintenance program meant making modifications to the resource provider (in this case inetd) and the resource requester (passwd client). The inetd had to be modified to export the resources it offered, which in this case meant exporting passwd. Since many programs rely on inetd it was potentially risky to modify it. Instead a program inetd.init was developed which essentially performed the register and exports that inetd would if it had been modified. When inetd.init is killed it withdraws the inetd services and deregisters before dying as inetd would.

The inetd.init program exports the passwdd program by providing the passwdd's properties to the TBRMS. One of the essential properties inetd.init provides is the resourcelnterface for the password daemon. The resourcelnterface includes information about where passwdd is running, which port it is associated with, and what protocol it is expecting to use with the password client program.

The password client program had to be modified to use the TBRMS for locating the password server program. To find a suitable password server the client provides the TBRMS with its resource requirements. In the case of the password client it was important to find a passwdd program that served the right domain and used the same protocol. The password clients resource requirements were: resourceName = passwdd and protocol = uwocsdTwistedEudora and serviceDomain = syslab.csd.uwo.ca. When a resource matching was found the password client was able to use the resourcelnterface to successfully interact with the password server program. 
The success of the TBRMS prototype helps show that the TBRMS design is a feasible mechanism for managing resources in a general heterogeneous computing environment.

\section{Concluding Remarks}

This paper was motivated by the need and importance of managing resources in distributed computing systems. We examined the requirements for resource management, particularly using the Trader concepts proposed by the ODP standards. We presented a design of a Trader-Based Resource Management System, consisting of an architecture and resource management service interfaces.

Our prototype implementation of a Trader-Based Resource Management System using the X.500 Directory as its information repository has been completed and we have just started using it for managing a variety of distributed system resources. The performance measurement on the current prototype show that the time between a client's request and the TBRMS's reply is on the order of a couple of seconds. While work can be done to optimize this time, it does show that using the TBRMS does not add a significant overhead to the client's performance.

We are also in the process of instrumenting the client resource management service interface onto distributed applications and services that may utilize the TBRMS. As we reported earlier in this paper, the X.500 Directory possesses many characteristics that are quite desirable for supporting the operation of the resource management system as well as for the modelling of the resources that are to be managed by it.

For future work, it has been suggested that X.500 might serve a useful purpose in facilitating the federation of Traders [13]. We plan to examine federating our TBRMSs using X.500. This is natural since we are already using X.500 in our TBRMS implementation. A main use of the TBRMS is being planned in the area of distributed systems management. We plan to integrated the TBRMS into the distributed systems management testbed being currently developed here at the University of Western Ontario [8].

Our hope is that our current and future work with the ODP Trader can be beneficial to the refinement of the Trader standard itself as well as to the potential users of the Trader in various computing environments.

\section{References}

[1] M. Bauer, P. Finnigan, J. Hong, J. Rolia, T. Teorey, and G. Winters. Reference Architecture for Distributed Systems Management. IBM Systems Journal, 33(3):426-444, September 1994.

[2] M. Bearman and K. Raymond. Federating Traders: An ODP Adventure. Proc. of the IFIP Workshop on Open Distributed Processing, Berlin, Germany, 1991.

[3] M. Bearman and K. Raymond. Contexts, Views and Rules: An Integrated Approach to Trader Contexts. Proc. of the International Conference on Open Distributed Processing, pages 153163, Berlin, Germany, September 1993.

[4] John Bloomer. Power Programming with RPC. O'Reilly \& Associates, Inc,, Sebastopol, CA, 1992. 
[5] CCITT. The Directory - Overview of Concepts, Models and Services, CCITT X.500 Series Recommendations. CCITT, December 1988.

[6] CCITT. The Directory - Overview of Concepts, Models and Services, Draft CCITT X.500 Series Recommendations. CCITT, December 1991.

[7] J. W. Hong, M. A. Bauer, and J. M. Bennett. Integration of the Directory Service in the Network Management Framework. Proc. of the Third International Symposium on Integrated Network Management, pages 149-160, San Francisco CA, April 1993.

[8] J. W. Hong, M. A. Bauer, and H. L. Lutfiyya. Design of the Distributed Systems Management Testbed. Technical Report, in preparation, Dept. of Computer Science, University of Western Ontario, 1994.

[9] J. Indulska, K. Raymond, and M. Bearman. A Type Management System for an ODP Trader. Proc. of the International Conference on Open Distributed Processing, pages 141152, Berlin, Germany, September 1993.

[10] ITU-TS. Basic Reference Model of Open Distributed Processing Part 1: Overview and Guide to the Use of the Reference Model. ITU-TS Rec X.901, ISO/IEC 10746-1, July 1992.

[11] ITU-TS. Draft ODP Trading Function. ITU-TS SG7.Q16 Draft Recommendation, July 1994.

[12] C. Popien and B. Hager. The ODP Trader Functionality Applied to the Integrated Road Transport Environment. Proc. of the Globecom'93, pages 1202-1206, Houston, TX, November 1993.

[13] C. Popien and B. Meyer. Federating ODP Traders: An X.500 Approach. Proc. of the ICC'93, Geneva, Switzerland, May 1993.

[14] A W. Pratten. Resource Management in a Distributed Computing Environment. MSc. Thesis, Dept. of Computer Science, University of Western Ontario, London, Ontario, Canada, September 1994.

[15] P. Putter and J. D. Roos. Relationships: Implementing Transparency in Distributed Management Systems. Proc. of the IEEE First International Workshop on Systems Management, pages 118-124, Los Angeles, CA, April 1993.

[16] C. J. Robbins and S. E. Kille. The ISO Development Environment: User's Manual Version 8.0. X-Tel Services Ltd., June 1992.

[17] A. Vogel, M. Bearman, and A. Beitz. Enabling Interworking of Traders. Proc. of the IFIP International Conference on Open Distributed Processing, Brisbane, Australia, February 1995.

[18] C. Weider, R. Wright, and E. Feinler. A Survey of Advanced Usages of X.500. Internet Draft, IETF DISI Working Group, October 1992.

[19] W. Yeong, T. Howes, and S. Hardcastle-Kille. Lightweight Directory Access Protocol. Internet Engineering Task Force OSI-DS Working Document 26, August 1992. 


\section{About the Authors}

A Warren Pratten received his BA in Geography, his BSc in Computer Science and his MSc in Computer Science from the University of Western Ontario in 1989, 1992 and 1994 respectively. $\mathrm{He}$ is currently working as a systems administrator in the department. His research interests include distributed computing and systems management. He can be reached via electronic mail at warren@esd. uwo.ca.

James W. Hong is a research associate and adjunct professor in the Department of Computer Science at the University of Western Ontario. He received his BSc and MSc from the University of Western Ontario in 1983 and 1985 respectively and his doctorate from the University of Waterloo in 1991. He is a member of the ACM and IEEE. His research interests include distributed computing, software engineering, systems and network management. He can be reached via electronic mail at jwkhong@csd. uwo.ca.

Michael A. Bauer is Chairman of the Department of Computer Science at the University of Western Ontario. He received his doctorate from the University of Toronto in 1978. He has been active in the Canadian and International groups working on the X.500 Standard. He is a member of the ACM and IEEE and is a member of the ACM Special Interest Group Board. His research interests include distributed computing, software engineering and computer system performance. He can be reached via electronic mail at bauer ${ }_{C s d}$. uwo.ca.

J. Michael Bennett is an associate professor in the Department of Computer Science at the University of Western Ontario. He received his doctorate from the University of Western Ontario in 1972. $\mathrm{He}$ has been active in the Canadian and International groups working on the X.500 standard. He is a member of the ACM and IEEE. His research interests include distributed computing, network management, computer system performance, communications and computer architecture.

Hanan L. Lutfiyya is an assistant professor of Computer Science at the University of Western Ontario. She received her B.S. in computer science from Yarmouk University, Irbid, Jordan in 1985, her M.S. from the University of Iowa in 1987, and her doctorate from the University of MissouriRolla in 1992. She is a member of the ACM and IEEE. Her research interests include distributed computing, formal methods in software engineering and fault tolerance. She can be reached via electronic mail at hananecsd. uwo.ca. 